\title{
A height based graphic method was more accurate than estimation based on external landmarks for determining depth of paediatric gastric tube insertion
}

\author{
Klasner AE, Luke DA, Scalzo AJ. Pediatric orogastric and nasogastric tubes: a new formula evaluated. Ann Emerg Med \\ 2002 Mar;39:268-72.

\section{QUESTION: Is a graphic method for determining depth of gastric tube insertion based on paediatric patient height more accurate than the traditional method based on measurements from the external landmarks of the nose or mouth, to the earlobe, to the xiphoid process (NEX method)?}

Source of funding: no external funding.

For correspondence Dr AE Klasner,

University of Alabama Birmingham, AL, USA. aklasner@peds.uab.edu

\section{Design}

Randomised \{allocation concealed\}*, blinded (outcome assessors), controlled trial with immediate follow up.

\section{Setting}

The emergency department of a university affiliated children's hospital in \{St Louis, Missouri,USA\}*.

Graphic method (based on patient height) $v$ NEX method for determining depth of paediatric gastric tube insertion

\begin{tabular}{lccc} 
Outcomes & Graphic & NEX & Difference $(95 \% \mathrm{Cl})$ \\
$\begin{array}{l}\text { Mean insertion distance from the centre of } \\
\text { the stomach }(\mathrm{cm})\end{array}$ & -1.12 & 1.31 & $2.43(1.33$ to 3.54$)$ \\
\hline $\begin{array}{l}\text { Mean absolute value of insertion distance } \\
\text { from the centre of the stomach }(\mathrm{cm})\end{array}$ & 1.26 & 2.60 & $1.34(0.50$ to 2.18$)$ \\
\hline
\end{tabular}

TNEX = measurement from the nose or mouth, to the earlobe, to the xiphoid process. Other abbreviations defined in glossary. Negative values indicate underinsertion and positive values indicate overinsertion.

\section{COMMENTARY}

Gastrointestinal intubation is a common paediatric procedure, but it is not without risk. Serious complications such as oesophageal perforation are rare, but deep or shallow insertions can also cause problems. Tubes passed too far into the stomach may coil or kink, and become obstructed. If a tube is not inserted far enough, or if the tip re-enters the oesophagus and stomach contents enter the lungs, pneumonia may develop.

In the study by Klasner $e t a l$, $x$ rays of 89 children aged 6 months to 18 years were examined to compare the merits of a nomogram based on height, with the traditional NEX measurement method. The centre of the stomach was regarded as the optimal location for the distal tip of the tube, and the results were reported in terms of deviation (either + or -) from this landmark. A trend towards overinsertion was noted for children in the NEX group ( $50 \%$ of insertions between +1 to $+4 \mathrm{~cm}$ from the centre of the stomach), whereas tubes of children in the graphic group fell slightly short $(50 \%$ of insertions between -1 to $-2 \mathrm{~cm}$ from the centre of the stomach). Greater variability of insertion distances was observed in the NEX group.

The randomisation of participants, stratification based on height, and blinding of assessors reviewing the $\mathrm{x}$ rays add strength to the study. However, the authors regarded the centre of the stomach as the only correct placement for an intragastric tube, when in practice, small deviations from the centre may not be clinically significant, particularly in adolescents. Although 3 tubes in the NEX group were found to be outside of the confines of the stomach, it was not stated whether these were underinserted or overinserted, or whether they were found in only one age group. This may have been an occasion when reporting subgroup results could be informative. Although the mean ages in the groups were similar, reporting variability of insertion distances by age bands may have been useful to facilitate judgment about the superiority of one method over the other.

The results of Klasner et al suggest that the graphic method may be a valid alternative to the traditional NEX method, but further work is needed to identify which, if any, patient groups would be most likely to benefit from this change of practice.

Jeanette Robertson, RN, MSc Deputy Director Western Australian Centre for Evidence Based Nursing and Midwifery King Edward/Princess Margaret Hospitals Subiaco, Western Australia, Australia
Patients

89 children who were 6 months to 18 years of age and needed gastric intubation in the emergency department. Exclusion criteria were previous oesophageal surgery, known congenital abnormalities of the oesophagus, or need for emergency placement of the gastric tube (eg, critically ill trauma and ingestion patients in which obtaining study consent might have delayed treatment). Data from 88 children $(99 \%)$ were included in the analysis (mean age $54 \mathrm{mo}, 61 \%$ boys).

\section{Intervention}

Children were stratified (tall, medium, and short) according to percentile height on the basis of standard growth curves and block randomised. 45 children were allocated to the graphic method, whereby depth of gastric tube insertion was determined from a nomogram based on height. 44 children were allocated to the NEX method, whereby depth of tube insertion was estimated by measuring the distance from the nose or mouth to the earlobe, to a point midway between the xiphoid process and the umbilicus. A physician specified the type and size of tube and whether it was nasogastric or orogastric. Nurses placed and secured the tubes and assessed correct placement by auscultation, instillation of air, and aspiration for gastric contents.

\section{Main outcome measures}

Average length of tube overinsertion or underinsertion $(0=$ perfect placement, a positive number $=$ overinsertion, and a negative number $=$ underinsertion) and insertion accuracy (ie, distance from the centre of the stomach) based on abdominal radiographs blindly assessed by 2 paediatric emergency physicians.

\section{Main results}

3 tubes, all in the NEX group, were found to be outside of the confines of the stomach. Tubes inserted using the graphic method had a smaller mean distance from the centre of the stomach compared with the NEX method (table) and showed less variability.

\section{Conclusion}

For paediatric gastric tube insertion, a graphic method based on height was more accurate than the standard method of measuring the distance from the nose or mouth, to the earlobe, to the xiphoid process for determining depth of tube insertion.

*Information provided by author. 